\title{
Bayesian parameter estimation of core collapse supernovae using gravitational wave simulations
}

\author{
Matthew C. Edwards ${ }^{1,2}$, Renate Meyer ${ }^{1}$ and Nelson \\ Christensen ${ }^{2}$ \\ ${ }^{1}$ Department of Statistics, University of Auckland, Auckland 1142, New Zealand \\ ${ }^{2}$ Physics and Astronomy, Carleton College, Northfield, Minnesota 55057, USA \\ E-mail: matthew.edwards@ligo.org, renate.meyer@auckland.ac.nz and \\ nchriste@carleton.edu
}

\begin{abstract}
Using the latest numerical simulations of rotating stellar core collapse, we present a Bayesian framework to extract the physical information encoded in noisy gravitational wave signals. We fit Bayesian principal component regression models with known and unknown signal arrival times to reconstruct gravitational wave signals, and subsequently fit known astrophysical parameters on the posterior means of the principal component coefficients using a linear model. We predict the ratio of rotational kinetic energy to gravitational energy of the inner core at bounce by sampling from the posterior predictive distribution, and find that these predictions are generally very close to the true parameter values, with $90 \%$ credible intervals $\sim 0.04$ and $\sim 0.06$ wide for the known and unknown arrival time models respectively. Two supervised machine learning methods are implemented to classify precollapse differential rotation, and we find that these methods discriminate rapidly rotating progenitors particularly well. We also introduce a constrained optimization approach to model selection to find an optimal number of principal components in the signal reconstruction step. Using this approach, we select 14 principal components as the most parsimonious model.
\end{abstract}

PACS numbers: $04.80 . \mathrm{Nn}, 02.70 . \mathrm{Uu}, 97.60 . \mathrm{Bw}, 95.85 . \mathrm{Sz}$ 


\section{Introduction}

In his general theory of relativity, Albert Einstein predicted the existence of gravitational waves (GWs) [1] - ripples in the fabric of spacetime caused by asymmetries in catastrophic and highly accelerated events throughout the cosmos. Though confirmed indirectly by observations of the binary pulsar PSR 1913 + 16 [2], GWs have not been directly detected by the global network of first generation detectors, such as Initial LIGO in the United States [3, 4, Virgo in Italy [5, 6], GEO 600 in Germany [7, and TAMA 300 in Japan [8].

The second generation of LIGO detectors, Advanced LIGO [9], are currently under construction and will likely operate as early as 2015 [10]. Advanced Virgo [11] should come on-line in 2016, while the Japanese KAGRA [12] detector will join the worldwide network later in the decade. The ten-fold improvement in sensitivity of these detectors [9, 10, 13], along with coherent analysis between observatories, will significantly improve the chances of detecting GWs from an astrophysical event in the Milky Way and neighbouring galaxies. It is therefore likely that direct detection of GWs will occur in the near future.

Potential sources of GWs include the inspiral of compact binary star systems (of neutron stars or black holes) followed by black hole formation [14], pulsars [15], rotating core collapse supernovae (CCSN) followed by protoneutron star formation [16], gammaray bursts [17], and cosmic string cusps [18].

Rotating CCSN are of particular interest in this paper. Like neutrinos, GWs are emitted deep in the core of a progenitor and propagate through the universe mostly unobscured by astrophysical objects between the source and a detector on Earth. GWs act like messengers, providing primary observables about the multi-dimensional core collapse dynamics and emission mechanisms. It is in this way that GW astronomy will open a new set of eyes to view the universe, complementing the conventional electromagnetic-type observations.

Coalescing binary star systems are the most promising source of detectable GWs [14, with an expected observation rate that could be as large as a few hundred events per year for Advanced LIGO [10, 19]. In contrast, the expected rate of CCSN in the Milky Way is around three per century [20. It is of great importance that appropriate data analysis techniques are in place so we do not miss an opportunity to detect these rare CCSN events.

The Bayesian statistical framework has proven to be a powerful tool for parameter estimation in astrophysical and cosmological settings [21]. Bayesian data analysis was first introduced to the GW community by Christensen and Meyer 22. Christensen and Meyer [23] then demonstrated the usefulness of the Gibbs sampler [24, 25] for estimating five physical parameters of coalescing binary signals. Christensen, Meyer, and Libson [26] then went on to show how a custom-built Metropolis-Hastings algorithm [24, 27, 28], a generalization of the Gibbs sampler, was a superior and more suited routine for eventual implementation into the LIGO Scientific Collaboration (LSC) algorithm library 
(LAL). Parameter estimation for compact binary inspirals has subsequently become more sophisticated in recent years (see for example [29, 30, 31, 32, 33, 34, 35]). Markov chain Monte Carlo (MCMC) routines for inferring the physical parameters of pulsars have also been developed [36, 37, 38].

Due to the analytical intractability and complex multi-dimensional nature of rotating core collapse stellar events, a significant amount of computational time must go into numerically simulating the gravitational waveforms. Unlike binary inspiral events, one cannot simply use template search methods for supernova burst events as it is computationally impossible to cover the entire signal parameter space. It is therefore important to find alternative parameter estimation techniques.

Summerscales et al [39] utilized the maximum entropy framework to deconvolve noisy data from multiple (coherent) detectors, with the goal of extracting a CCSN GW signal. Inference on amplitude and phase parameters was conducted using cross correlation between the recovered waveform and the set of competing waveforms from the Ott et al [16] catalogue. A match was defined as the model with the maximum cross correlation to the recovered waveform.

Heng [40] first proposed a principal component analysis (PCA) approach to simplify the problem by reducing a given supernova waveform catalogue space down to a small number of basis vectors. Röver et al [41] extended this approach and created a novel Metropolis-within-Gibbs sampler [24] to reconstruct test signals from the Dimmelmeier et al catalogue [42] in noisy data using a principal component regression (PCR) model with random effects and unknown signal arrival time. They then attempted to exploit the structure of the posterior principal component (PC) coefficients with a simple $\chi^{2}$ measure of distance to determine which catalogue waveform best matched the injected test signal. Although the Bayesian reconstruction method showed much promise, extraction of the underlying physical parameters had limited success.

Logue et al [43] used nested sampling [44] to compute Bayesian evidence for PCR models under three competing supernova mechanisms - neutrino, magnetorotational, and acoustic mechanisms. Each supernova mechanism has a noticeably distinct gravitational waveform morphology, and the method was successful at correctly inferring a large majority of injected signals. They found that for signals embedded in simulated Advanced LIGO noise, the magnetorotational mechanism could be distinguished to a distance of up to $10 \mathrm{kpc}$, and the neutrino and acoustic mechanisms up to $2 \mathrm{kpc}$.

Abdikamalov et al [45] generated a new CCSN waveform catalogue and applied matched filtering [46] to infer total angular momentum to within $\pm 20 \%$ for rapidly rotating cores. Slowly rotating cores had errors up to $\pm 35 \%$. Along with matched filtering, they employed the Bayesian model selection method presented in [43] to illustrate that under certain assumptions of the rotation law, the next generation of GW detectors (Advanced LIGO, Advanced Virgo, and KAGRA), could also extract information about the degree of precollapse differential rotation. The two methods worked particularly well for rapidly rotating cores.

In this paper we present an alternative approach to parameter estimation for 
rotating CCSN. Using the Abdikamalov et al catalogue [45], we fit a Bayesian PCR model to reconstruct a GW signal in noisy data. Initially, the signal arrival time is assumed to be known, and PC coefficients are sampled directly from the posterior distribution. We extend the model to incorporate an unknown signal arrival time and construct a Metropolis-within-Gibbs MCMC sampler (as done in [41]). We then use the posterior means of the PC coefficients to fit the known physical parameters on (using a linear model), and sample from the posterior predictive distribution to make probabilistic statements about the ratio of rotational kinetic energy to gravitational energy of the inner core at bounce $\beta_{i c, b}$. We apply two supervised learning algorithms — naïve Bayes classifier (NBC) and $k$-nearest neighbour $(k-\mathrm{NN})$ - to classify the closest level of precollapse differential rotation $A$. We also introduce a constrained optimization approach to model selection and attempt to find an optimal number of PCs for the Bayesian PCR model.

The paper is organized as follows: in section 2 we describe the simulated GW data catalogue used in our analysis; section 3 introduces the statistical models and methods applied; results of our analysis are presented in section 4; and a discussion of our findings and future directions are provided in section 5 .

\section{Gravitational wave data}

The waveforms used in this paper are the two-dimensional numerical axisymmetric general-relativistic hydrodynamic rotating core collapse and bounce supernova simulations generated by Abdikamalov et al [45]. Based on findings that GW signals are essentially independent of the progenitor zero age main sequence (ZAMS) mass by Ott et al [47], a single presupernova progenitor model (the 12- $M_{\odot}$ at ZAMS solar-metallicity progenitor model from [48]) was adopted. The cylindrical rotation law from [16] was also assumed.

The GW catalogue was partitioned into a base catalogue (BC), and a test catalogue (TC). The BC contains $l=92$ signals with five levels of precollapse differential rotation $A$ (where higher values of $A$ represent weaker differential rotation), a grid of values for initial central angular velocity $\Omega_{c}$, and a grid of values for the ratio of rotational kinetic energy to gravitational energy of the inner core at bounce $\beta_{i c, b}$ (since $\beta_{i c, b}$ is a function of $\Omega_{c}$ for a fixed progenitor structure). Each signal in the BC was generated using the microphysical finite-temperature Lattimer-Swesty (LS) equation of state (EOS) [49], parametrized deleptonization scheme from [42], and neutrino leakage scheme from [47]. As well as varying $A, \Omega_{c}$, and $\beta_{i c, b}$, the TC contains 47 signals with differing EOS and deleptonization parametrizations $Y_{e}(\rho)$. Specifically, some test signals were generated using the Shen et al EOS [50], or an increase/decrease in $Y_{e}(\rho)$ parametrization by $\sim 5 \%$. The values of $\Omega_{c}$ and $\beta_{i c, b}$ in the $\mathrm{TC}$ are in the same parameter space as those in the $\mathrm{BC}$, but with an alternative grid. The object of our analysis is to predict the physical parameters $\left(\beta_{i c, b}\right.$ and $A$ ) of the signals in the TC using information gleaned about signals in the $\mathrm{BC}$. 
The signals were initially sampled at $100 \mathrm{kHz}$ and subsequently downsampled by a rational factor to $16384 \mathrm{~Hz}$ - the sampling rate of the Advanced LIGO detectors. Downsampling by a rational factor essentially involved two steps: upsampling by an integer factor via interpolation and then applying a low-pass filter to eliminate the high frequency components necessary to avoid aliasing at lower sampling rates; and downsampling by an integer factor to achieve the desired sampling rate [51]. The resampled data was zero-bufferred to ensure each signal was the same length, $N=16384$, which corresponded to $1 \mathrm{~s}$ of data at the Advanced LIGO sampling rate. Each signal was then aligned so that the first negative peak (not necessarily the global minimum), corresponding to the time of core bounce, occurred halfway through the time series.

In this analysis, the source of a $\mathrm{GW}$ emission is assumed to be optimally oriented (perpendicular) to a single interferometer. Each signal is linearly polarized with zero cross-polarization.

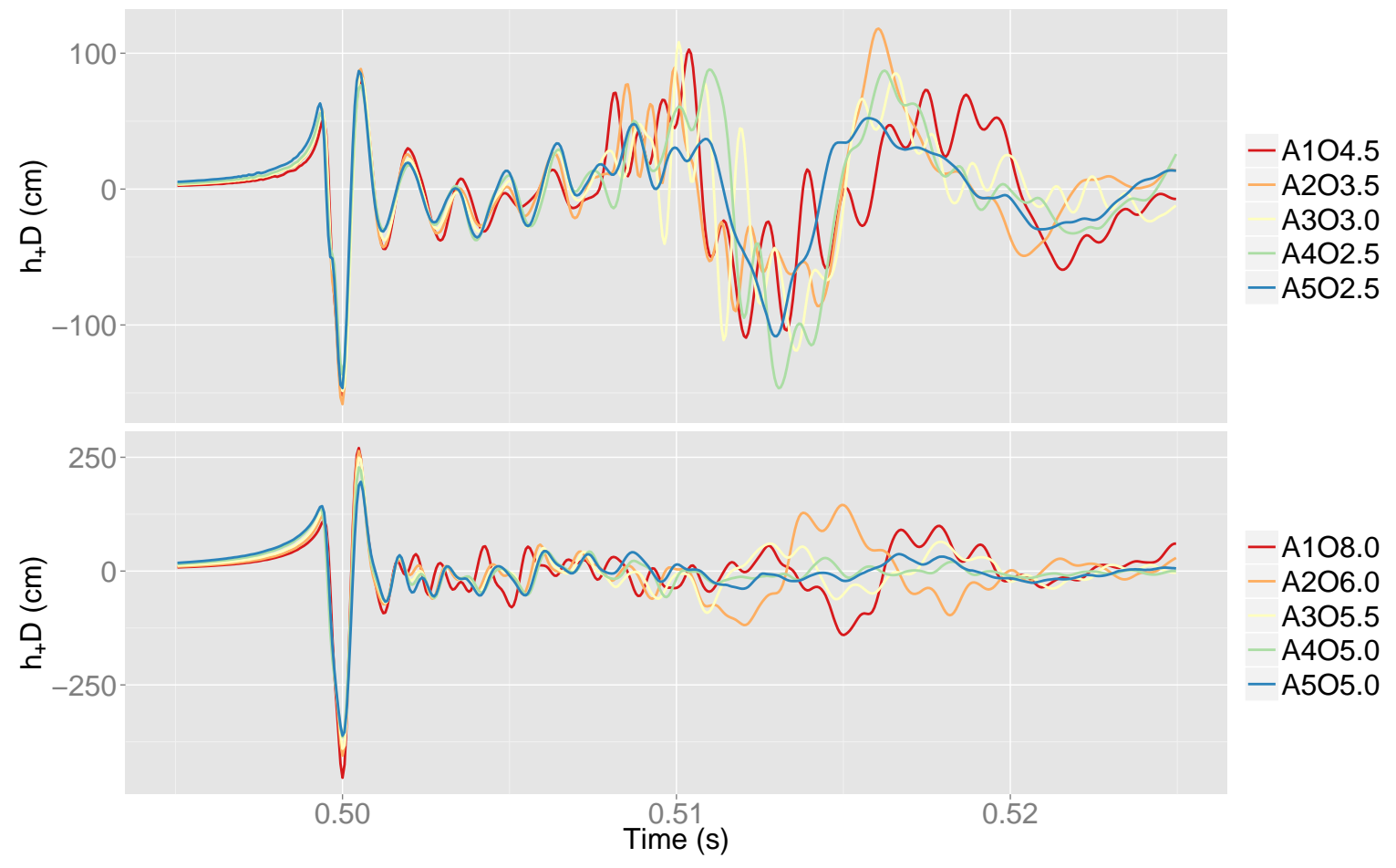

Figure 1: A snapshot of the Abdikamalov et al [45] catalogue. The top panel shows the GW strain (scaled by source distance) for five models with different levels of precollapse differential rotation (from strongest differential rotation $A 1$ to weakest $A 5$ ), each with $\beta_{i c, b} \sim 0.03$ (i.e., slowly rotating progenitors). The bottom panel is the same, but for rapidly rotating progenitors with $\beta_{i c, b} \sim 0.09$.

We can see a general waveform morphology in figure 1. During core collapse, there is a slow increase in GW strain until the first local maximum is reached (before $0.5 \mathrm{~s}$ ). This is followed by core bounce, where the strain rapidly decreases towards a local minimum (at $0.5 \mathrm{~s}$ ). This corresponds to the time when the inner core expands at bounce. After this, there is a period of ring-down oscillations. For slowly rotating 
progenitors, we see in the top panel of figure 1 that the GW strain is essentially the same during collapse and bounce and only differs during ring-down. For the rapidly rotating progenitors presented in the bottom panel of figure 1, larger precollapse differential rotation results in: a smaller local maximum during core collapse; a more negative local minimum during core bounce; and a larger first ring-down peak. Because of these patterns, Abdikamalov et al [45] concluded that inferences about precollapse differential rotation could in principal be made for rapidly rotating cores.

The data analyzed are CCSN GW signals injected in coloured Gaussian noise using the Advanced LIGO noise curve with one-sided power spectral density (PSD), $S_{1}(f)$. The data is then Tukey windowed to mitigate spectral leakage. Rather than fixing source distance to $10 \mathrm{kpc}$ (as done in [45]), this analysis assumes a fixed signal-to-noise ratio (SNR) of $\rho=20$. $\mathrm{SNR}$ is defined as

$$
\rho=\sqrt{4 \sum_{j} \frac{\frac{\Delta_{t}}{N}\left|\tilde{y}_{j}\right|^{2}}{S_{1}\left(f_{j}\right)}},
$$

where $\tilde{y}_{j}, j=1,2, \ldots, N$, are the Fourier transformed data, $\Delta_{t}$ is the distance between two consecutive time points, and $f_{j}, j=1,2, \ldots, N$, are the Fourier frequencies. As done in [41], $S_{1}(f)$ is estimated a priori by averaging 1000 empirical periodograms from identically simulated Advanced LIGO noise. This corresponds to a realistic scenario where the noise spectrum must be estimated as well.

Although supernovae from the Milky Way will not produce SNRs as small as $\rho=20$, we choose this value to illustrate that our methods are robust at lower SNRs.

\section{Methods and models}

\subsection{Bayesian inference}

Bayesian inference requires three pivotal quantities. The likelihood function $p(\mathbf{z} \mid \boldsymbol{\theta})$ is the probability density function (PDF) of the data $\mathbf{z}$, conditional on the random vector

of model parameters $\boldsymbol{\theta}$. The prior $p(\boldsymbol{\theta})$ is the PDF of the model parameters, that takes into account all of the information known about $\boldsymbol{\theta}$ before the data is observed. The posterior $p(\boldsymbol{\theta} \mid \mathbf{z})$ is the updated PDF of model parameters after the data is observed. These quantities are related via Bayes' theorem

$$
\begin{aligned}
p(\boldsymbol{\theta} \mid \mathbf{z}) & =\frac{p(\boldsymbol{\theta}) p(\mathbf{z} \mid \boldsymbol{\theta})}{m(\mathbf{z})} \\
& \propto p(\boldsymbol{\theta}) p(\mathbf{z} \mid \boldsymbol{\theta}),
\end{aligned}
$$

where $m(\mathbf{z})=\int p(\boldsymbol{\theta}) p(\mathbf{z} \mid \boldsymbol{\theta}) \mathrm{d} \boldsymbol{\theta}$ is the marginal likelihood and is treated as a normalizing constant since it is independent of $\boldsymbol{\theta}$. That is, the posterior is proportional to the prior multiplied by the likelihood.

Posterior sampling can be performed directly if the posterior PDF has a closed analytical form. Otherwise, MCMC techniques are a useful work-around. The key 
building blocks in MCMC simulations are the Gibbs sampler [25] and the MetropolisHastings algorithm [27, 28]. We use a combination of the two - the so-called Metropoliswithin-Gibbs sampler - in this study. For a detailed account of Bayesian inference and MCMC algorithms, refer to [24].

\subsection{Model 1: Bayesian PCR with known signal arrival time}

We aim to first reduce the dimension of the BC by a PCA, or equivalently a singular value decomposition (SVD) as suggested by Heng [40]. Each BC waveform is represented as a linear combination of orthonormal basis vectors, where the projection of the data onto the first basis vector has maximum variance, the projection onto the second basis vector has second highest variance, and so on. By considering only projections on the first $d<l$ basis vectors, the so-called $d$ PCs, a parsimonious representation of the catalogue signals in $d$ dimensions is achieved that preserves as much of the information of the original $\mathrm{BC}$ as possible.

Once PCA is conducted, the first $d$ PCs are treated as the explanatory variables of a linear model. The data analyzed is a time series vector $\mathbf{y}$ of length $N$ and decomposes into additive signal and noise components. Let $\tilde{\mathbf{y}}$ be the Fourier transformed data vector of length $N$ and let $\tilde{\mathbf{X}}$ be the $N \times d$ design matrix, whose columns are the Fourier transformed mean-centered $\mathrm{PC}$ vectors from the $\mathrm{BC}$. The frequency domain linear model is

$$
\tilde{\mathbf{y}}=\tilde{\mathbf{X}} \boldsymbol{\alpha}+\tilde{\boldsymbol{\epsilon}}
$$

where $\boldsymbol{\alpha}$ is the vector of PCR coefficients and $\tilde{\boldsymbol{\epsilon}}$ is the Fourier transformed coloured zero-mean Gaussian noise vector whose variance terms are proportional to the a priori known one-sided power spectral density $S_{1}(f)$. That is,

$$
\sigma_{f_{j}}^{2}=\frac{N}{4 \Delta_{t}} S_{1}\left(f_{j}\right)
$$

Due to Hermitian symmetry, the frequency domain data vector $\tilde{\mathbf{y}}$ contains only the non-redundant real and imaginary components and is therefore the same length as the time domain vector $\mathbf{y}$. Conversion between time and frequency domains is conducted using a fast Fourier transform (FFT).

The likelihood for the Bayesian PCR model with known signal arrival time is

$$
p(\tilde{\mathbf{y}} \mid \boldsymbol{\alpha}) \propto \exp \left(-2 \sum_{j=1}^{N} \frac{\frac{\Delta_{t}}{N}\left(\tilde{y}_{j}-(\tilde{\mathbf{X}} \boldsymbol{\alpha})_{j}\right)^{2}}{S_{1}\left(f_{j}\right)}\right) .
$$

Assuming flat (Uniform $(-\infty, \infty)$ ) priors on $\boldsymbol{\alpha}$, the posterior distribution for the PC coefficients is

$$
\mathrm{P}(\boldsymbol{\alpha} \mid \tilde{\mathbf{y}})=\mathrm{N}(\boldsymbol{\mu}, \boldsymbol{\Sigma})
$$


where

$$
\begin{aligned}
& \boldsymbol{\Sigma}=\left(\tilde{\mathbf{X}}^{\prime} \mathbf{D}^{-1} \tilde{\mathbf{X}}\right)^{-1} \\
& \boldsymbol{\mu}=\boldsymbol{\Sigma} \tilde{\mathbf{X}}^{\prime} \mathbf{D}^{-1} \tilde{\mathbf{y}}
\end{aligned}
$$

and $\mathbf{D}=\operatorname{diag}\left(\sigma_{f_{j}}^{2}\right)$ is the diagonal covariance matrix of individual variances for the noise component. This multivariate normal distribution can be sampled directly with no MCMC required.

Noninformative priors were chosen for this model. It was important to keep the data and prior knowledge separate and distinct, and to avoid using information from the waveform catalogue for both purposes. As the only data available for analysis were the generated GWs, we assumed complete prior ignorance on all model parameters.

\subsection{Model 2: Bayesian PCR with unknown signal arrival time}

The Bayesian PCR model presented in the previous section assumed a known signal arrival time. The precise arrival time of a GW signal to an interferometer will generally not be known in practice, and must therefore be included as an additional unknown parameter in the statistical model.

Let $T$ be a cyclical time shift representing the unknown signal arrival time, and let $\tilde{\mathbf{X}}_{T}$ be the Fourier transformed design matrix $\tilde{\mathbf{X}}$ shifted by lag $T$, such that the Fourier transformed PCs are aligned with the Fourier transformed data vector, $\tilde{\mathbf{y}}$. This transformation can be done directly in the frequency domain as a phase shift by multiplying the columns of $\tilde{\mathbf{X}}$ by $\exp (-2 \pi \mathrm{i} f T)$.

We build on the Bayesian signal reconstruction model presented in [41], although our primary goal is inferring the physical parameters of a supernova progenitor and not signal reconstruction.

Using the same reasoning described in the previous section, we assume flat priors on $\boldsymbol{\alpha}$ and $T$. The likelihood for the Bayesian PCR model with unknown signal arrival time is

$$
p(\tilde{\mathbf{y}} \mid \boldsymbol{\alpha}, T) \propto \exp \left(-2 \sum_{j=1}^{N} \frac{\frac{\Delta_{t}}{N}\left(\tilde{y}_{j}-\left(\tilde{\mathbf{X}}_{T} \boldsymbol{\alpha}\right)_{j}\right)^{2}}{S_{1}\left(f_{j}\right)}\right) .
$$

For a given time shift $T$, the conditional posterior distribution for the $\mathrm{PC}$ coefficients $\boldsymbol{\alpha} \mid T$ is

$$
\mathrm{P}(\boldsymbol{\alpha} \mid T, \tilde{\mathbf{y}})=\mathrm{N}\left(\boldsymbol{\mu}_{T}, \boldsymbol{\Sigma}_{T}\right)
$$

where

$$
\begin{aligned}
& \boldsymbol{\Sigma}_{T}=\left(\tilde{\mathbf{X}}_{T}^{\prime} \mathbf{D}^{-1} \tilde{\mathbf{X}}_{T}\right)^{-1}, \\
& \boldsymbol{\mu}_{T}=\boldsymbol{\Sigma}_{T} \tilde{\mathbf{X}}_{T}^{\prime} \mathbf{D}^{-1} \tilde{\mathbf{y}} .
\end{aligned}
$$

To estimate $\boldsymbol{\alpha}$ and $T$, we construct a Markov chain whose stationary distribution is the posterior distribution of interest using Metropolis-within-Gibbs sampler [24]. This is 
essentially a Gibbs sampler that alternates between the full set of conditional posterior distributions $P(\boldsymbol{\alpha} \mid T, \tilde{\mathbf{y}})$ and $P(T \mid \boldsymbol{\alpha}, \tilde{\mathbf{y}})$. The former can be sampled directly using equation (11), and the latter requires a random walk Metropolis step, hence the name Metropolis-within-Gibbs.

After initialization, step $i+1$ in the Metropolis-within-Gibbs algorithm is:

(i) Directly sample the conditional posterior of $\alpha_{i+1} \mid T_{i}$ using equation (11);

(ii) Propose $T_{*}$ from $t_{\nu}\left(T_{i}, \zeta^{2}\right)$ and accept $T_{i+1}=T_{*}$ with the Metropolis acceptance probability

$$
r=\min \left(1, \frac{p\left(T_{*} \mid \boldsymbol{\alpha}, \tilde{\mathbf{y}}\right)}{p\left(T_{i} \mid \boldsymbol{\alpha}, \tilde{\mathbf{y}}\right)}\right) .
$$

Otherwise reject and set $T_{i+1}=T_{i}$.

A $t$-distribution was chosen as the proposal distribution for the algorithm. It has a similar (symmetrical) shape to the normal distribution but has heavier tails and an additional degrees-of-freedom parameter, $\nu$. The heavier tails of the $t$-distribution results in bolder and more robust proposals than the normal distribution, ensuring the algorithm does not get stuck in local modes [24]. The degrees-of-freedom parameter was set to $\nu=3$, which is the smallest integer that yields a distribution with finite variance. The proposal for $T_{i+1}$ is centered on $T_{i}$, and has scale parameter $\zeta^{2}$ that is initially and arbitrarily set to 0.05 , and subsequently automatically tuned during the algorithm to ensure good mixing and acceptance rates.

\subsection{Posterior predictive distribution}

For each of the $l=92$ signals in the BC and $m=47$ signals in the TC, we fit both Bayesian PCR models, with $d$ PCs (where the choice of $d$ is explained below). We then construct an $l \times(d+1)$ design matrix $\mathbf{A}$ whose rows are the posterior means of the $d$ $\mathrm{PC}$ coefficients, plus an intercept term, for each of the $l$ signals in the BC. The primary goal is to exploit the posterior PC coefficient space to make inferences on the physical parameters of rotating core collapse stellar events in the TC. We accomplish this by fitting a linear model with the known physical parameters from the $\mathrm{BC}$ as the response variable on the design matrix A using

$$
\boldsymbol{\xi}=\mathrm{A} \gamma+\boldsymbol{\delta},
$$

where $\boldsymbol{\xi}$ is the vector of known continuous physical parameters, $\boldsymbol{\gamma}$ is the vector of regression coefficients, and $\boldsymbol{\delta}$ is an error term. The error term is assumed to come from an independent and identically distributed normal distribution with zero mean and variance $\sigma^{2}$. Predictions using the posterior predictive distribution are the primary interest in this analysis, and not the model parameters themselves.

Assuming the convenient noninformative prior distribution that is uniform on $(\gamma, \log \sigma)$, the posterior predictive distribution for a normal linear model is a multivariate $t$-distribution and can be sampled from directly with no MCMC [24]. The formula is

$$
\mathrm{P}(\check{\boldsymbol{\xi}} \mid \boldsymbol{\xi})=t_{l-d}\left(\check{\mathbf{A}} \hat{\boldsymbol{\gamma}}, s^{2}\left(I+\check{\mathbf{A}} \mathbf{V}_{\boldsymbol{\xi}} \check{\mathbf{A}}^{\prime}\right)\right) \text {, }
$$


where $\check{\boldsymbol{\xi}}$ is the vector of outcomes we wish to predict (i.e., the physical parameters from signals in the TC), $\check{\mathrm{A}}$ is the $m \times(d+1)$ matrix whose rows are the posterior means of the signals in the TC (and an intercept term) from the Bayesian PCR step, $I$ is the $m \times m$ identity matrix, and

$$
\begin{aligned}
\mathbf{V}_{\boldsymbol{\xi}} & =\left(\mathbf{A}^{\prime} \mathbf{A}\right)^{-1}, \\
\hat{\boldsymbol{\gamma}} & =\mathbf{V}_{\boldsymbol{\xi}} \mathbf{A}^{\prime} \boldsymbol{\xi}, \\
s^{2} & =\frac{1}{l-d}(\boldsymbol{\xi}-\mathbf{A} \hat{\boldsymbol{\gamma}})^{\prime}(\boldsymbol{\xi}-\mathbf{A} \hat{\boldsymbol{\gamma}}) .
\end{aligned}
$$

\subsection{Deviance information criterion and constrained optimization}

The deviance is defined as $D=-2 \log p(\mathbf{z} \mid \boldsymbol{\theta})$ where $p(\mathbf{z} \mid \boldsymbol{\theta})$ is the likelihood of a statistical model, and $\boldsymbol{\theta}$ is the vector of model parameters. The deviance information criterion (DIC) is a Bayesian model comparison technique and a generalization of Akaike information criterion (AIC) for hierarchical models [52]. DIC is defined as

$$
\begin{aligned}
\mathrm{DIC} & =\bar{D}+p_{D} \\
& =2 \bar{D}-D(\overline{\boldsymbol{\theta}}),
\end{aligned}
$$

where $\bar{D}$ is the mean deviance from posterior samples, $p_{D}$ is the effective number of parameters, and $D(\overline{\boldsymbol{\theta}})$ is the deviance evaluated at the posterior means of the parameters. When comparing competing statistical models, the lowest DIC is preferred. $\bar{D}$ is a measure of fit, and $p_{D}$ is a measure of model complexity used to penalize models with too many parameters. Equation (20) therefore illustrates how DIC incorporates Occam's razor, allowing one to select a parsimonious model, balancing between fit and complexity. Equation (21), on the other hand, provides a simple method for computing DIC. $\bar{D}$ is calculated by evaluating the deviance for each of the stored model parameters $\boldsymbol{\theta}$ that have been sampled from their joint posterior PDF, and then taking the average. $D(\overline{\boldsymbol{\theta}})$ is calculated by finding the posterior mean of each of the model parameters $\overline{\boldsymbol{\theta}}$ and then evaluating the deviance.

DIC is the preferred model comparison technique in this analysis. A popular alternative, Bayes factors, would require computing the marginal likelihood from equation (2), which involves multi-dimensional integration over a large number of parameters. Numerical techniques such as nested sampling [44] can be used to derive the marginal likelihood but these methods require significant computational time and power. On the other hand, DIC is easily computed from posterior samples. Another benefit of using DIC over Bayes factors is that improper priors (which we have assumed in this analysis) do not violate any conditions of use. Bayes factors, on the other hand, are no longer applicable when improper priors are used.

The choice of the number of PCs has been arbitrary in most of the supernova GW parameter estimation literature and this number has usually been $d=10$ (see for example [41, 45]). We propose a method for selecting the optimal choice of $d$ based on careful analysis of the DIC for competing models and constrained optimization. Since 
PCs are ordered by the total amount of variation they make up in the data set, PCA provides a convenient ordering system for nested modelling. Let $M_{d}, d \in\{1,2, \ldots, 92\}$, represent the set of possible PCR models, where $d$ is the number of explanatory variables. The models are nested such that $M_{1}$ has one explanatory variable (PC1), $M_{2}$ has two explanatory variables (PC1 and $\mathrm{PC} 2$ ), and so on.

For each of the $l=92$ signals in the BC (injected in Advanced LIGO noise), all of the models $M_{d}, d \in\{1,2, \ldots, 92\}$, are fitted and then compared using DIC. The model with the lowest DIC is the best fit to the data. However, models with an absolute difference in DIC of $\lesssim 5$ are generally taken to be indistinguishable from one another [52] and so to prevent over-fitting, we propose a constrained optimization routine, where we select the smallest $d$ such that the difference in DIC between $M_{d}$ and the model with the minimum DIC is less than 5. More specifically, let $M_{\min }$ be the model with the minimum DIC, then find $d$ such that

$$
\underset{d}{\operatorname{argmin}}\left\{\operatorname{DIC}\left(M_{d}\right)-\operatorname{DIC}\left(M_{\min }\right)<5\right\} .
$$

We employ this routine for each of the $l=92 \mathrm{BC}$ signals, and look at the distribution of $M_{d}$ 's over all signals. The median of this distribution seems a prudent choice for a general-purpose number of PCs since these distributions tend to be skewed. It is important to note here that we cannot choose a different value for $d$ for each signal when implementing these models as this would lead to a very sparse design matrix $\mathbf{A}$ when sampling from the posterior predictive distribution.

We refer the reader to figure 2 in the results section of this paper for an example of this method in action.

\subsection{Nä̈ve Bayes classifier}

The NBC [53] is a common supervised learning algorithm and discriminant method used to group objects into a discrete set of classes based on a set of features. The algorithm requires a training set of objects with known groupings and observed features. Once the algorithm has learnt from the training set, each object in a test set (containing a set of observed features and potentially unknown classes) is assigned to the group that it has the highest probability of belonging to.

The "Bayes" component of the method refers to Bayes' theorem

$$
p(c \mid \mathbf{u}) \propto p(c) p(\mathbf{u} \mid c)
$$

where $c \in C$ is the class that an object could belong to, and $\mathbf{u}$ are the features we wish to exploit to classify the object. That is, given some observed features $\mathbf{u}$, what is the posterior probability of an object belonging to class $c$ ?

The "naïve" component refers to the assumption of conditional independence of the model features $\mathbf{u}=\left(u_{1}, u_{2}, \ldots, u_{d}\right)$. This assumption implies the joint PDF $p(\mathbf{u} \mid c)$ can be factorized as the product of marginal distributions

$$
p(\mathbf{u} \mid c)=\prod_{i=1}^{d} p\left(u_{i} \mid c\right),
$$


and so equation 23 becomes

$$
p(c \mid \mathbf{u})=p(c) \prod_{i=1}^{d} p\left(u_{i} \mid c\right) .
$$

Given class $c$, each feature $\left(u_{1}, u_{2}, \ldots, u_{d}\right)$ is assumed to be independently normally distributed. The model parameters are approximated using the relative frequencies from the training set. The class prior probabilities $p(c)$ are specified as the number of objects in class $c$ in the training set divided by the total number of objects. Objects are grouped into the class that yields the highest posterior probability. This is known as the maximum a posteriori (MAP) decision rule.

\section{7. $k$-nearest neighbour}

An alternative machine learning algorithm to the $\mathrm{NBC}$ is the $k$-NN [53], which uses a measure of "closeness" between objects rather than a probabilistic framework. We choose $k=1$, meaning that an object in the test set is assigned to the class of its single nearest neighbour in the training set. Ties in distance are settled at random.

The definition of closeness in this context depends on the choice of metric. As commonly used in the literature [53], a Euclidean distance is assumed. For any object with features $\mathbf{u}=\left(u_{1}, u_{2}, \ldots, u_{d}\right)$ in the test set, the $k$-NN algorithm finds the object with features $\mathbf{v}=\left(v_{1}, v_{2}, \ldots, v_{d}\right)$ in the training set that minimizes the Euclidean distance

$$
\operatorname{distance}(\mathbf{u}, \mathbf{v})=\sqrt{\sum_{i=1}^{d}\left(u_{i}-v_{i}\right)^{2}},
$$

and then assigns $\mathbf{u}$ to the class of $\mathbf{v}$.

\section{Results}

\subsection{Model selection}

An important statistical task is to select a prudent number of model dimensions whilst incorporating Occam's razor into the decision making process. More specifically, one needs to balance model fit against complexity to ensure there is no over-fitting. In the context of PCA, the decision is usually made based on the amount of variation the first $d$ PCs contribute to the data set (i.e., analyzing Scree plots). This approach is arbitrary and deals specifically with dimension reduction, but not Occam's razor. We propose an alternative approach, involving DIC and constrained optimization.

We analyze the change in DIC as model dimensionality increases. Figure 2 illustrates DIC as a function of model dimensionality for signal A1O2.5 from the Abdikamalov et al catalogue [45]. This is the typical shape of the DIC curve for all signals in the BC and a good visual aid of Occam's razor in action. There tends to be a sharp decrease in DIC as the model dimension increases at the beginning, where model 
fit is improving. DIC flattens out and then reaches a minimum, where there is the best balance between fit against complexity. After this, there is a slow rise in DIC as the model dimension increases and becomes too complex.

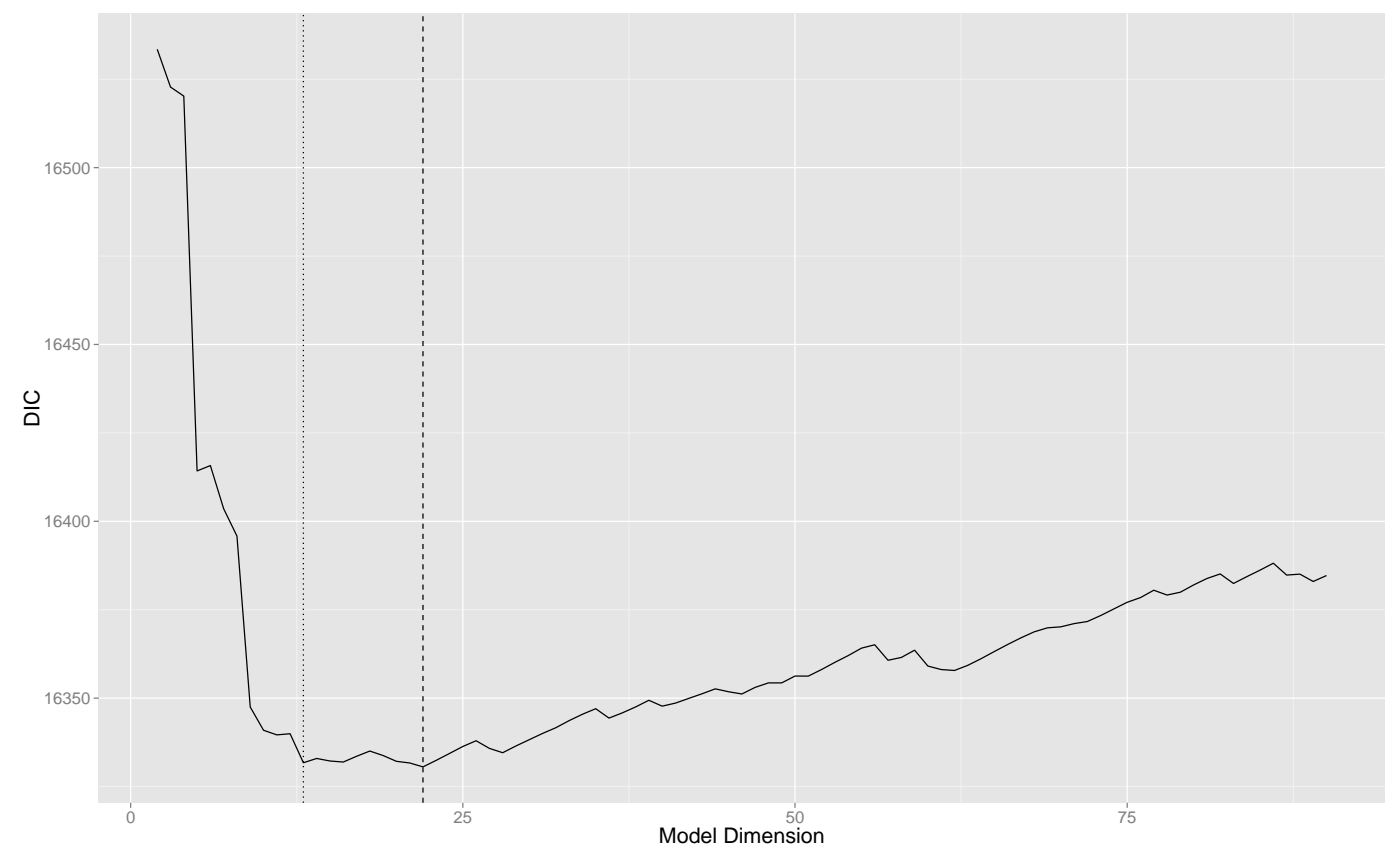

Figure 2: DIC as a function of model dimensionality for model $A 1 O 2.5$ from the Abdikamalov et al catalogue [45]. The dashed vertical line to the right represents the model with the minimum DIC $\left(M_{\min }=M_{22}\right)$. The dotted vertical line to the left represents the model dimension after constrained optimization $\left(M_{d}=M_{13}\right)$.

The flat basin around the global minimum in figure 2 is of particular interest. Since models with an absolute difference in DIC of less than 5 are essentially indistinguishable, it is sensible to select the model with the smallest number of dimensions in this region to prevent over-fitting. For signal $A 1 O 2.5$, we see a significant decrease in model dimensionality from $M_{\min }=M_{22}$ to $M_{d}=M_{13}$. The choice of $d$ for this particular signal is $d=13$.

It is important to note that $d$ will differ between GW signals but we must only choose one general-purpose value of this. We therefore conduct the proposed constrained optimization model selection method on all of the $l=92 \mathrm{BC}$ signals and take the median of the distribution of $d$ 's as the general-purpose $d$. We prefer the median to the mean as our central measure as it is more robust against outliers. 


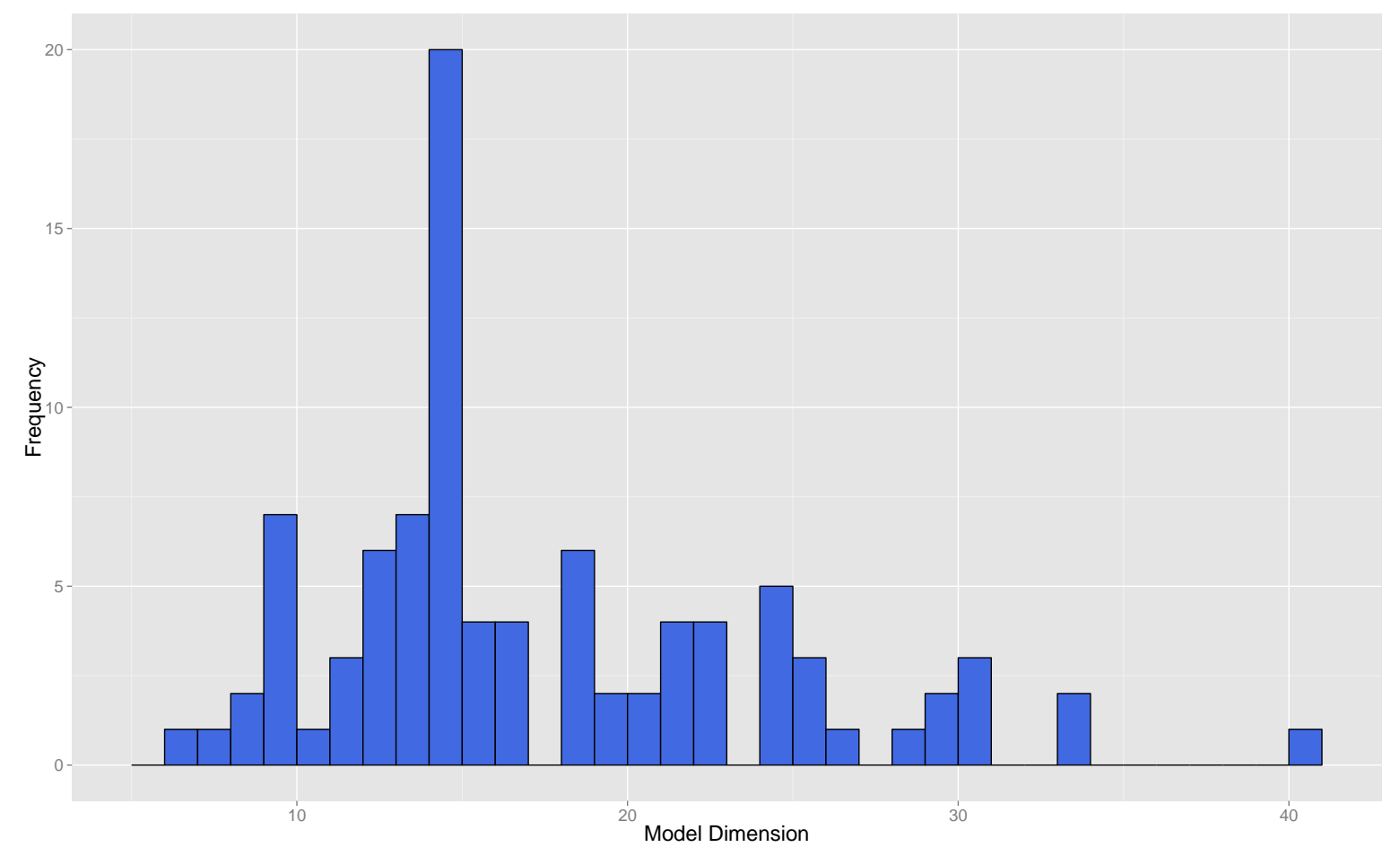

Figure 3: Distribution of model dimensionality for all $l=92$ signals in the BC under our constrained optimization routine.

The histogram in figure 3 shows the distribution of $d$ for all $l=92$ signals in the BC. It is highly skewed to the right, with a median (and mode) of 14 PCs and mean of 17 PCs. We choose $d=14$ based on the median of this distribution, and use this number of explanatory variables in both Bayesian PCR models. We choose this as the model that minimizes the risks of both over-fitting and under-fitting.

4.2. Inferring the ratio of rotational kinetic energy to gravitational energy of the inner core at bounce, $\beta_{i c, b}$

We injected each of the $l=92 \mathrm{BC}$ and $m=47$ TC signals in Advanced LIGO noise (SNR $\rho=20$ ) and fitted the two Bayesian PCR models with $d=14$ PCs. We then regressed the known value of $\beta_{i c, b}$ on the posterior means of the $\mathrm{BC}$ signals from these models and sampled from the posterior predictive distribution of the TC signals. Figures 44 show these predictions of $\beta_{i c, b}$. The true value from the TC (red triangle) is compared with the predicted value (blue circle) and uncertainty is measured using $90 \%$ credible intervals (black lines). Figures 4 and 5 assume a known signal arrival time. $T$ is unknown for figures 6 and 7 . The change in background gradient for figures 4 and 6 represents the varying precollapse differential rotation model $A$ for signals with LS EOS and standard $Y_{e}(\rho)$ parametrization. For figures 5 and 7 , the background shade represents GW signals (from a precollapse differential rotation model $A 1$ ) with a Shen EOS, or increase/decrease in $Y_{e}(\rho)$ of $\sim 5 \%$. $\beta_{i c, b}$ is scaled up by a factor of 100 in these plots. 


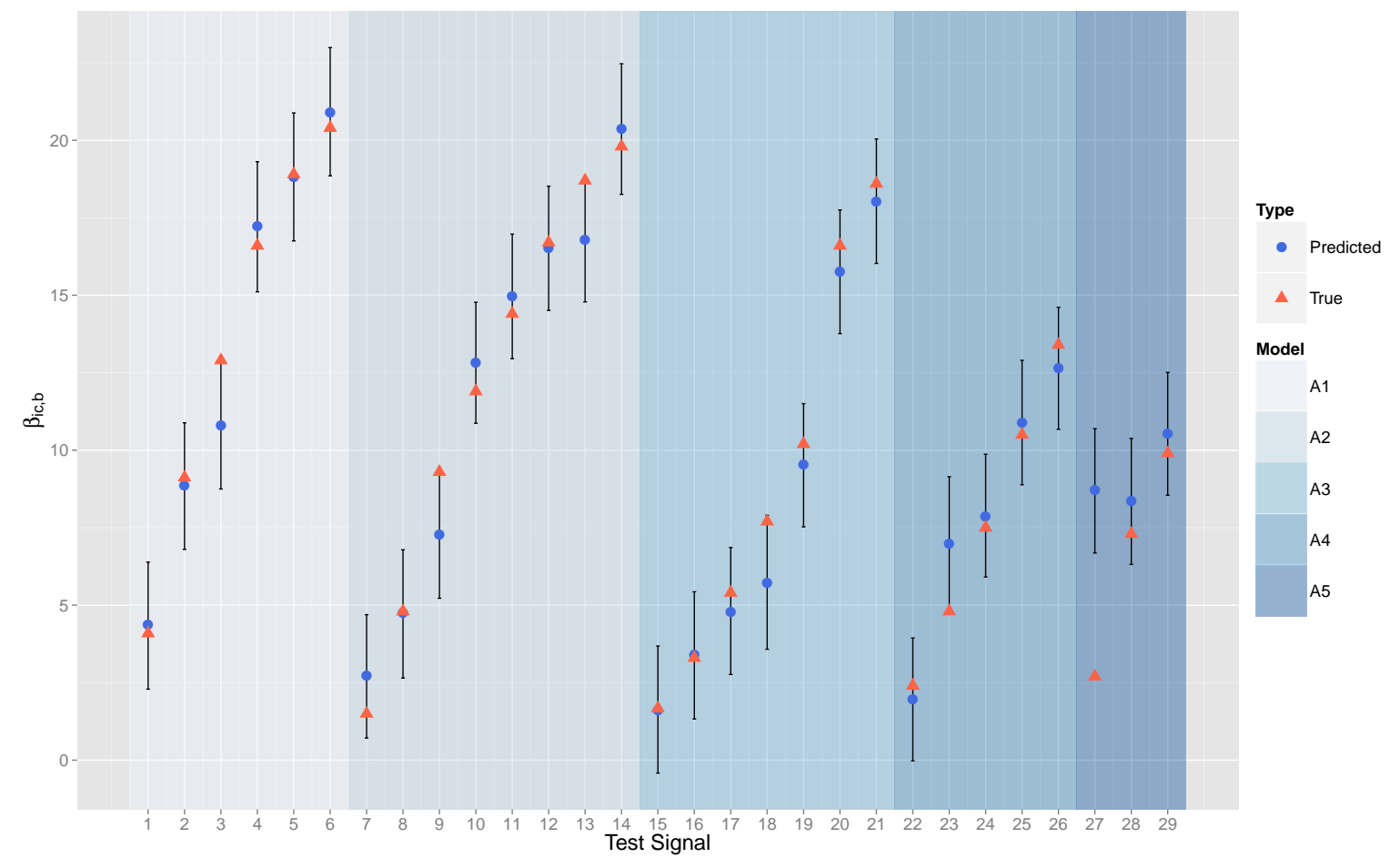

Figure 4: $90 \%$ credible intervals of $\beta_{i c, b}$ for the 29 test signals with the LS EOS and standard $Y_{e}(\rho)$ parametrization. $T$ is known.

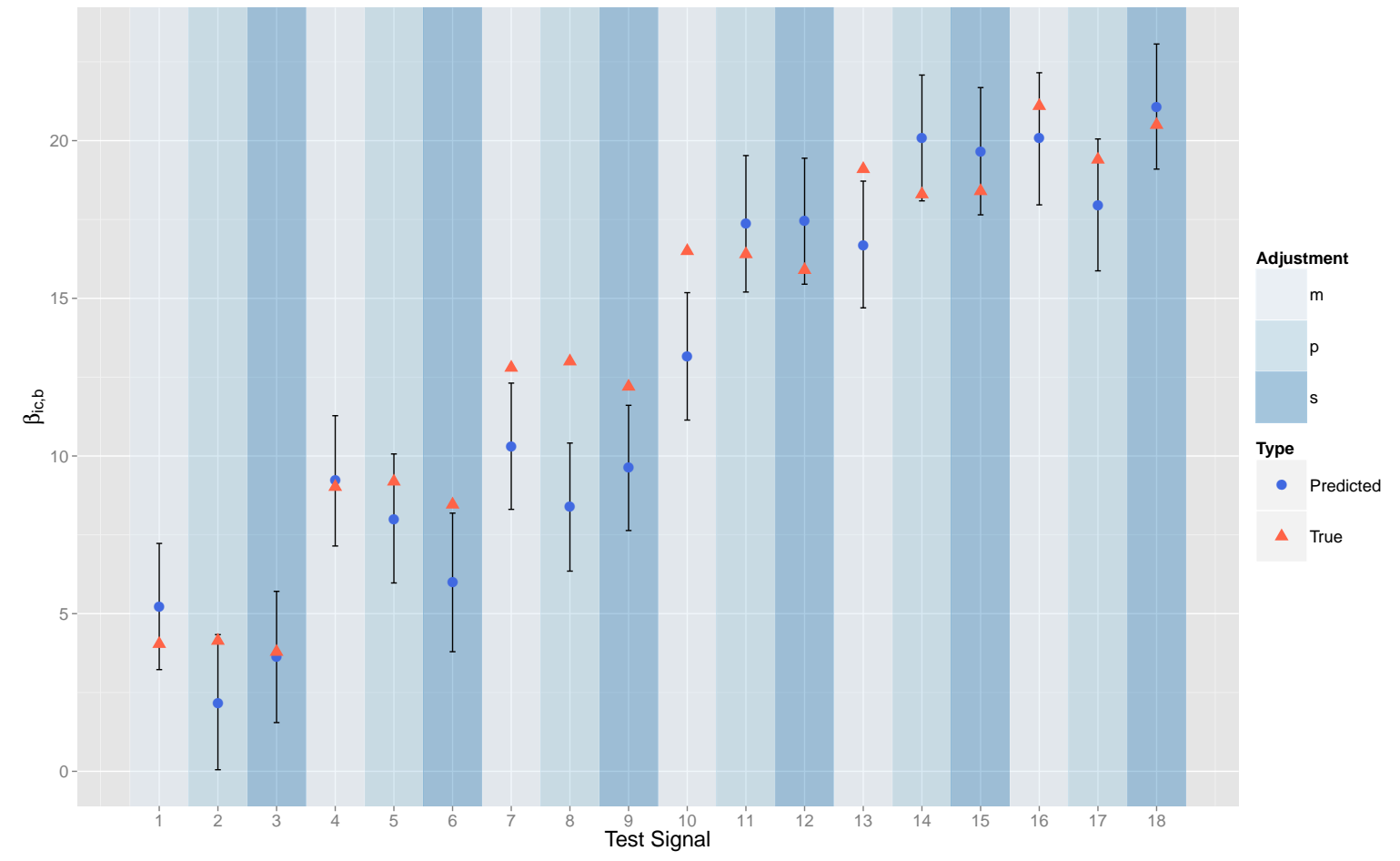

Figure 5: $90 \%$ credible intervals of $\beta_{i c, b}$ for the 18 test signals with varying EOS and $Y_{e}(\rho)$ parametrization. Note that $m$ refers to an increase in $Y_{e}(\rho)$ of $5 \%, p$ refers to a decrease in $Y_{e}(\rho)$ of $5 \%$, and $s$ refers to the Shen EOS. $T$ is known. 


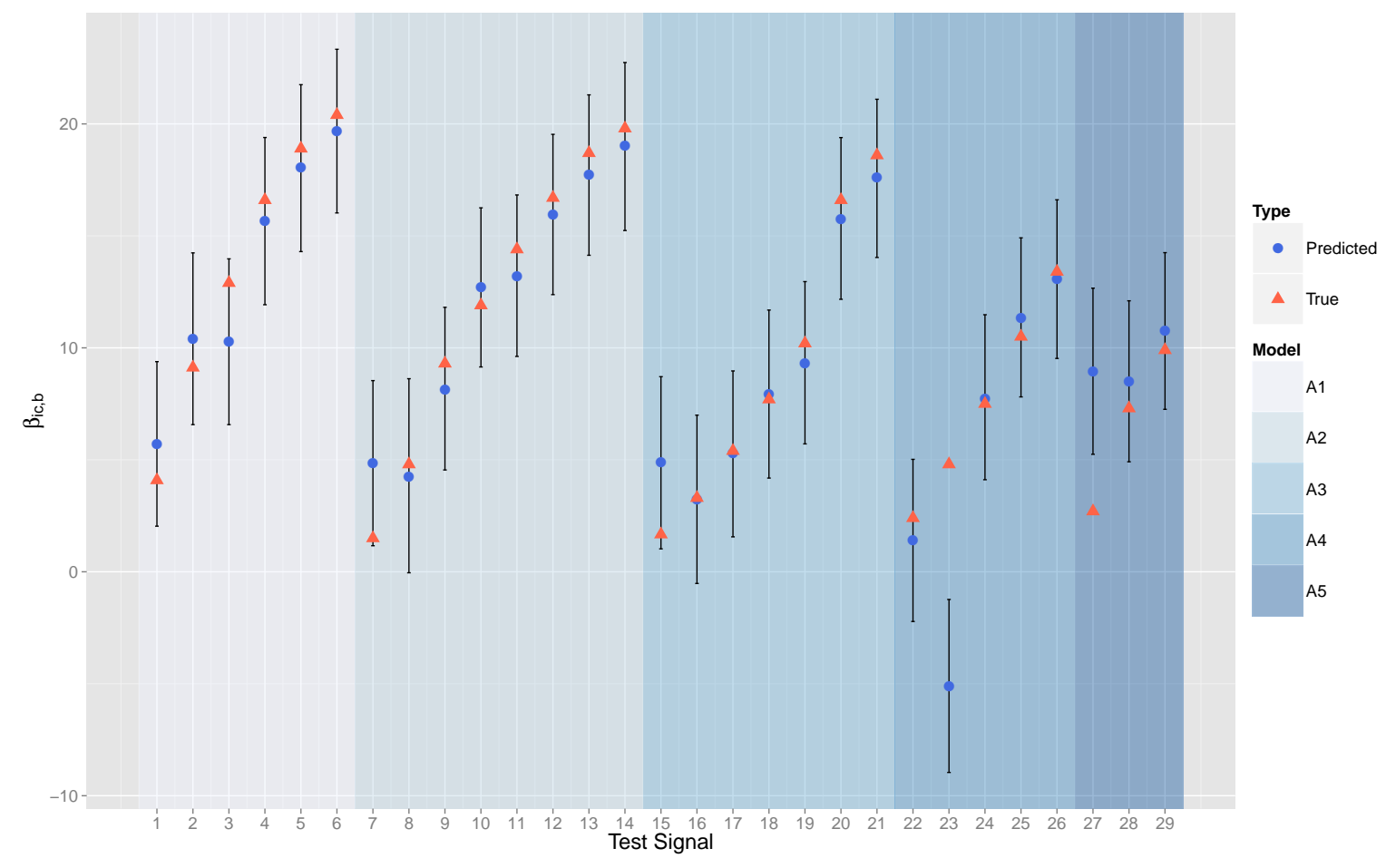

Figure 6: $90 \%$ credible intervals of $\beta_{i c, b}$ for the 29 test signals with the LS EOS and standard $Y_{e}(\rho)$ parametrization. $T$ is unknown.

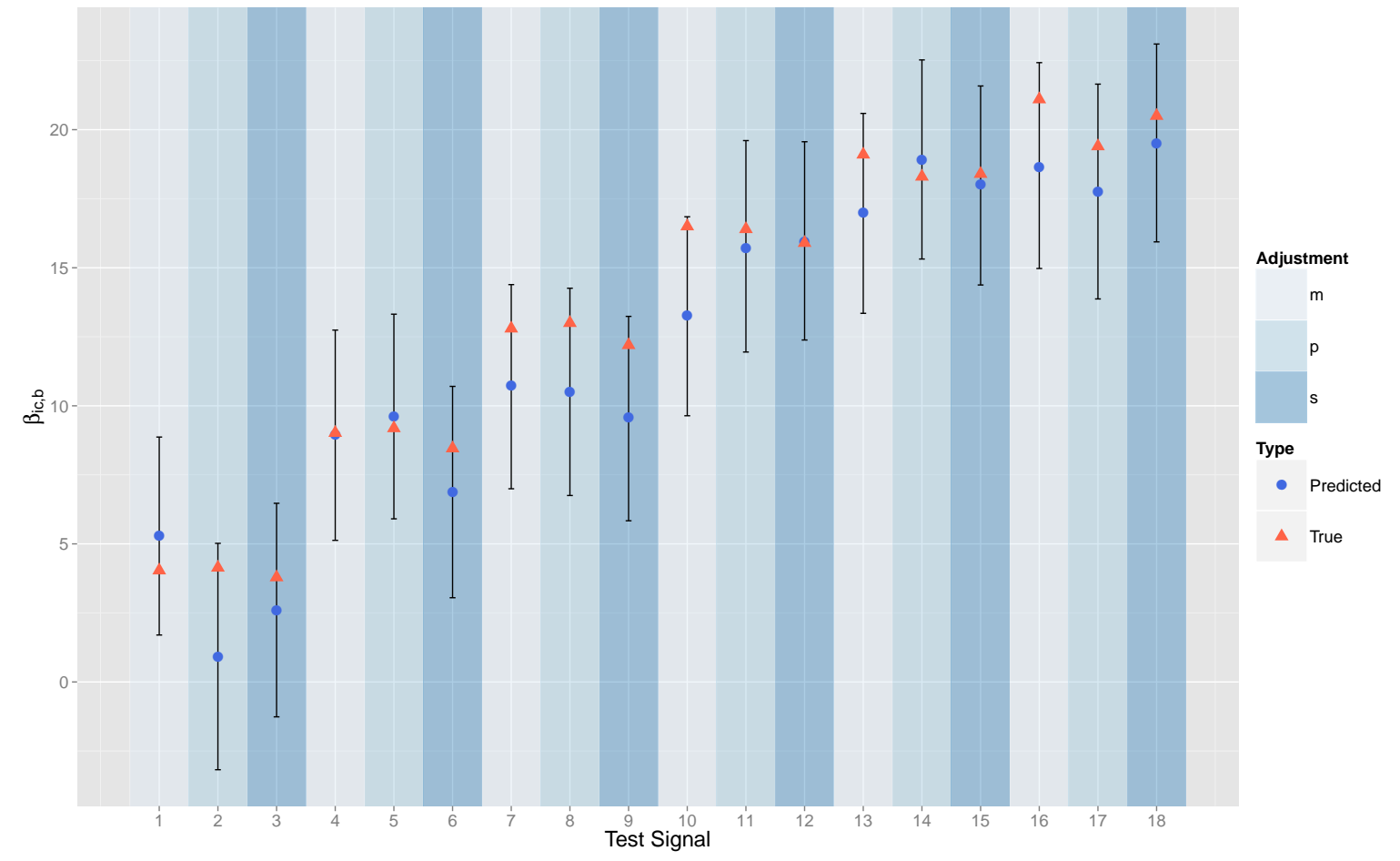

Figure 7: $90 \%$ credible intervals of $\beta_{i c, b}$ for the 18 test signals with varying EOS and $Y_{e}(\rho)$ parametrization. Note that $m$ refers to an increase in $Y_{e}(\rho)$ of $5 \%, p$ refers to a decrease in $Y_{e}(\rho)$ of $5 \%$, and $s$ refers to the Shen EOS. $T$ is unknown. 
We yield accurate predictions of $\beta_{i c, b}$ for most of the test signals in figure 4. Signal 27 ( $A 503.25$ from the catalogue) is an outlier and comes from a slowly rotating core with uniform rotation. It is likely an outlier due to the strong stochastic components in the GW signal from prompt postbounce convection [45]. The true values of $\beta_{i c, b}$ are on the boundary of the $90 \%$ credible intervals for signals 3 (A1O10.25), 9 (A2O6.25), 19 (A3O5.25), and 23 (A4O3.25), but there is no distinguishable pattern between these signals. The credible intervals are relatively small, at approximately four units (times $10^{-2}$ ) long. This means that it is particularly easy to distinguish $\beta_{i c, b}$ between GW signals.

The length of credible interval widens by a factor of $\sim 1.5$ when changing from known to unknown signal arrival time. Incorporating an unknown time shift increases the uncertainty of the PC coefficients since the MCMC algorithm draws $\boldsymbol{\alpha} \mid T$. That is, conditioning on an uncertain $T$ creates additional uncertainty for $\boldsymbol{\alpha}$. However, predictions are still accurate in most cases. We see in figure 6, that signal 27 (A5O3.25) is an outlier again. Signal $23(A 4 O 3.25)$ is another outlier with credible interval on the negative side of the number line. This is an absurd and physically impossible range for a strictly positive variable, and is a consequence of the fact that the priors can only constrain the linear model parameters $\left(\gamma, \sigma^{2}\right)$. More specifically, we could not put priors on the response variable of physical parameters $\boldsymbol{\xi}$ to constrain the predicted physical parameters $\check{\xi}$. A similarity that this signal has with the other outlier is that it comes from a slowly rotating core with weak differential rotation.

Our methods work reasonably well when varying the EOS and deleptonization parametrization, although we underestimate some signals with moderate rotation in figure 5. Three of these signals come from an increase of $Y_{e}(\rho)$ parametrization, one from a decrease of $Y_{e}(\rho)$ parametrization, and two from the Shen EOS. When incorporating an unknown time shift in figure 7 , the uncertainty of $T$ increases and covers the true parameters. The increase in the width of credible interval makes it more difficult to distinguish $\beta_{i c, b}$ between signals.

We can conclude that the methods employed in this study are moderately sensitive to uncertainties in $Y_{e}(\rho)$ and EOS. It was found that a GW signal has relatively weak dependence on the nuclear EOS by [42]. We showed in an unpublished study [54] that we could correctly identify between the LS and Shen EOS for $50 \%$ of the signals in the Dimmelmeier et al [42] waveform catalogue using model comparison techniques. Note that $21 \%$ were incorrectly identified and $29 \%$ unidentified. It could therefore be useful to incorporate EOS as an additional unknown that we wish to infer in future statistical analyses.

The results presented assume a SNR of $\rho=20$. To test robustness, we trialled the analysis on SNRs of $\rho=50$ and $\rho=100$, which are more realistic levels for detecting CCSN events in the Milky Way. Our predictions and credible intervals of $\beta_{i c, b}$ were the same, regardless of the SNR. This can be attributed to using only the posterior means of the PC coefficients in constructing design matrix $\mathbf{A}$, and not the full spread of the posterior distributions. This therefore removes uncertainty due to LIGO noise and 
signal reconstruction when predicting $\beta_{i c, b}$ from the posterior predictive distribution.

\subsection{Classifying the precollapse differential rotation, A}

Precollapse differential rotation is treated as a categorical variable with five different levels in this analysis. We define the set of classes $C=\{A 1, A 2, A 3, A 4, A 5\}$ and apply the $\mathrm{NBC}$ and $k$-NN supervised learning algorithms to extract precollapse differential rotation from each of the signals in the TC. The model features $\mathbf{u}$ are the posterior means of the PC coefficients from the Bayesian PCR models ( $\overline{\boldsymbol{\alpha}}$ for the training set and

$\overline{\check{\alpha}}$ for the test set). The goal of this analysis is to let both algorithms learn from the training set to discriminate GW signals in the test set.

Table 1: Percentage of signals in the TC with correctly identified precollapse differential rotation $A$ using NBC and $k$-NN.

\begin{tabular}{lcccc}
\hline & \multicolumn{2}{c}{ Known $T(\%)$} & \multicolumn{2}{c}{ Unknown $T(\%)$} \\
\cline { 2 - 5 } Differential Rotation, $A$ & NBC & $k$-NN & NBC & $k$-NN \\
\hline$A 1$ & 83 & 83 & 83 & 83 \\
- Standard & 67 & 50 & 67 & 50 \\
$-\uparrow Y_{e}(\rho)$ & 67 & 83 & 83 & 100 \\
$-\downarrow Y_{e}(\rho)$ & 33 & 17 & 0 & 17 \\
- Shen EOS & 50 & 75 & 50 & 50 \\
$A 2$ & 43 & 57 & 29 & 57 \\
$A 3$ & 0 & 80 & 20 & 80 \\
$A 4$ & 33 & 33 & 0 & 33 \\
$A 5$ & & & & \\
\hline
\end{tabular}

Table 1 shows the percentage of signals in the TC that have a correctly identified level of $A$ using NBC and $k$-NN. We compare how the methods work when using $\overline{\boldsymbol{\alpha}}$ and $\bar{\alpha}$ from data with known and unknown signal arrival times.

The results between models with known and unknown signal arrival times are quite similar. The standard GWs from class $A 1$ are discriminated well by both algorithms. The decrease (and to some degree, the increase) in $Y_{e}(\rho)$ parametrization did not affect the algorithms' abilities to discriminate. Both algorithms performed particularly poorly for the Shen EOS test signals, which illustrates that $A$ is sensitive to the EOS. This is in line with the findings from [45].

The $k$-NN generally performs better than the NBC for GW signals with weak to moderate differential rotation $(A 3, A 4, A 5)$. This could be attributed to our choice in prior classes for the NB method. Since models with stronger differential rotation are more populated in the $\mathrm{BC}$, they have a higher prior probability than those with weaker differential rotation. 


\section{Discussion}

We have presented a Bayesian framework for inferring the physical parameters of CCSN from GW data. We have shown that with a SNR of $\rho=20$ and optimal orientation of detector to source, we can extract $\beta_{i c, b}$ with reasonable levels of uncertainty for the majority of injected test signals. Both of the Bayesian PCR models presented in this paper worked well. The level of uncertainty increased when incorporating an unknown signal arrival time into the model, but this is no surprise as PC coefficients are conditioned on the signal arrival time for that model. Further, we found that our methods were moderately sensitive to varying $\operatorname{EOS}$ and $Y_{e}(\rho)$ parametrizations, and predictions are generally good.

The chosen measure of uncertainty in this analysis was the $90 \%$ credible interval. A great benefit of the Bayesian framework is the probabilistic interpretation of credible intervals, enabling one to make statements such as, "with probability $0.9, \beta_{i c, b}$ is between $2.5 \times 10^{-2}$ and $6.5 \times 10^{-2} . "$

A true strength of the methods presented in this paper is their generality. We initially applied these techniques to the Dimmelmeier et al catalogue [42] as a proof of concept and then easily transferred to the Abdikamalov et al catalogue [45]. In this study we sampled $\beta_{i c, b}$ from its posterior predictive distribution. This method could however be conducted on any continuous variable of physical interest. Although not presented here, predictions of the initial central angular velocity $\Omega_{c}$ were comparable to what we found with $\beta_{i c, b}$.

Choosing to only use the posterior means of the PC coefficients $\overline{\boldsymbol{\alpha}}$ in the construction of the design matrix $\mathbf{A}$ removed some of the variability due to LIGO noise and signal reconstruction. The uncertainty from the Bayesian PCR modelling step therefore does not flow onto the posterior predictive sampling step. A more realistic case would be to incorporate this uncertainty through an errors-in-variables model, which is commonly used when there are measurement errors in the explanatory variables of a regression model. We plan to explore this in a future study. However, a benefit of our method was that predictions were essentially independent of SNR (at least for $\rho \geq 20$ ).

An important task in Bayesian analysis is specifying the prior PDF to describe our beliefs about model parameters before observing the data. We wanted to avoid using information from the waveform catalogue as both data and prior knowledge. It is in this light that we believe the waveform catalogue should be used only as data, and assume complete prior ignorance on all of the model parameters.

We applied the NBC and $k$-NN algorithm to extract precollapse differential rotation. We found that results were comparable between known and unknown signal arrival times. The $k$-NN algorithm generally performed better than the NBC under the assumptions made. In future work, we plan to investigate how the choice of prior for the NBC affects classification, as well as exploring different metrics such as the Mahalanobis distance (which takes correlations of the data into account) for the $k$-NN. We are also investigating an alternative classification routine, Bayesian ordinal probit regression. 
We introduced a constrained optimization approach to model selection that allowed us to select an appropriate number of PCs for the Bayesian PCR models. To our knowledge, this is the first attempt at doing so. Techniques such as reversible jump MCMC (RJMCMC) [55] have been utilized in GW data analysis contexts [56]. RJMCMC could prove to be a useful and more sophisticated approach than the method presented in the current study. Although our method required a lot of parallel computing, we found it to be a novel solution to the model selection problem.

Our analysis assumed optimal orientation of a GW source to a single interferometer. As presented in [30, 31] for compact binary inspiral signals, we plan to extend the methods presented in this study to a network of detectors. This is an important generalization as one can triangulate the position of a GW source using coherent data from multiple detectors. The ability to locate a GW source would allow astronomers to compare and verify whether there was a true astrophysical event or a glitch with electromagnetic observations.

\section{Acknowledgments}

We thank Ik Siong Heng for a thorough reading of the script, Ernazar Abdikamalov for providing us with the waveform catalogue and supplementary materials, Christian D. Ott for helpful discussions, and the New Zealand eScience Infrastructure (NeSI) for their high performance computing facilities and support. NC's work is supported by NSF grant PHY-1204371. This paper has been given LIGO Document Number P1400034.

\section{References}

[1] Einstein A 1916 Approximative integration of the field equations of gravitation Sitzungsberichte Preußischen Akademie der Wissenschaften 1916 (part 1) 688-96

[2] Taylor J and Weisburg J 1989 Further experimental tests of relativistic gravity using the binary pulsar PSR $1913+16$ Astrophys. J. 345 434-50

[3] Abramovici A et al 1992 LIGO: the laser interferometer gravitational-wave observatory Science $256325-33$

[4] Abbott B et al 2009 LIGO: the laser interferometer gravitational-wave observatory Rep. Prog. Phys. 72076901

[5] Caron B et al (the Virgo Collaboration) 1997 The Virgo interferometer Class. Quantum Grav. 14 $1461-9$

[6] Accadia $\mathrm{T}$ et al (the Virgo Collaboration) 2012 J. Instrum. 7 P03012

[7] Grote H (for the LIGO Scientific Collaboration) 2010 The GEO 600 status Class. Quantum Grav. 27084003

[8] Ando M (for the TAMA Collaboration) 2005 Current status of the TAMA300 gravitational-wave detector Class. Quantum Grav. 22 S881-9

[9] Harry G M (for the LIGO Scientific Collaboration) 2010 Advanced LIGO: the next generation of gravitational wave detectors Class. Quantum Grav. 27084006

[10] Aasi J et al (LIGO-Virgo Scientific Collaboration) 2013 Prospects for localization of gravitational wave transients by the Advanced LIGO and Advanced Virgo observatories Preprint arXiv:1304.0670 gr-qc] 
[11] Accadia T et al 2012 Advanced Virgo technical design report VIR-0128A-12 https://tds.egogw.it/ql/?c=8940

[12] Somiya K 2012 Detector configuration of KAGRA - the Japanese cryogenic gravitational-wave detector Class. Quantum Grav. 29124007

[13] Smith J R (for the LIGO Scientific Collaboration) 2009 The path to the enhanced and advanced LIGO gravitational-wave detectors Class. Quantum Grav. 26114013

[14] Thorne, K S 1987300 Years of Gravitation ed S W Hawking and W Israel (Cambridge: Cambridge University Press) pp 330-58

[15] Cutler C 2002 Gravitational waves from neutron stars with large toroidal B-fields Phys. Rev. D 66084025

[16] Ott C D, Burrows A, Livne E and Walder R 2004 Gravitational waves from axisymmetric rotating stellar core collapse Astrophys. J. 600 834-67

[17] Meszaros P 2006 Gamma-Ray Bursts Rep. Prog. Phys. 69 2259-322

[18] Damour T and Vilenkin A 2005 Gravitational radiation from cosmic (super)strings: bursts, stochastic background, and observational windos Phys. Rev. D $\mathbf{7 1} 063510$

[19] Abbott B (the LIGO Scientific Collaboration) 2005 Search for gravitational waves from galactic and extra-galactic binary neutron stars Phys. Rev. D 72082001

[20] Adams S M, Kochanek C S, Beacom J F, Vagins M R and Stanek K Z 2013 Observing the next galactic supernova Preprint arXiv:1306.0559v2[astro-ph.HE]

[21] Loredo T J 1992 Statistical Challenges in Modern Astronomy ed E D Fiegelson and G J Babu (New York: Springer) pp 275-97

[22] Christensen N and Meyer R 1998 Markov chain Monte Carlo methods for Bayesian gravitational radiation data analysis Phys. Rev. D 58082001

[23] Christensen N and Meyer R 2001 Using Markov chain Monte Carlo methods for estimating parameters with gravitational radiation data Phys. Rev. D 64022001

[24] Gelman A, Carlin J B, Stern H S, Dunson D B, Vehtari A and Rubin D B 2013 Bayesian Data Analysis 3rd edition (Boca Raton, FL: Chapman \& Hall / CRC)

[25] Geman S and Geman D 1984 Stochastic relaxation, Gibbs distributions, and the Bayesian restoration of images IEEE Trans. Patt. Anal. Mach. Intell. 6 721-41

[26] Christensen N, Meyer R and Libson A 2004 A Metropolis-Hastings routine for estimating parameters from compact binary inspiral events with laser interferometric gravitational radiation data Class. Quantum Grav. 21 317-30

[27] Metropolis N, Rosenbluth A W, Rosenbluth MN, Teller A H and Teller E 1953 Equation of state calculations by fast computing machines J. Chem. Phys. 21 1087-92

[28] Hastings W K 1970 Monte Carlo sampling methods using Markov chains and their applications Biometrika 57 97-109

[29] Röver C, Meyer R and Christensen N 2006 Bayesian inference on compact binary inspiral gravitational radiation signals in interferometric data $C Q G \mathbf{2 3} 4895-906$

[30] Röver C, Meyer R and Christensen N 2007 Coherent Bayesian inference of binary inspirals using a network of interferometric gravitational wave detectors Phys. Rev. D $\mathbf{7 5} 062004$

[31] Röver C, Meyer R, Guidi G M, Viceré A and Christensen N 2007 Coherent Bayesian analysis of inspiral signals Class. Quantum Grav. 24 S607-15

[32] Raymond V, van der Sluys M V, Mandel I, Kalogera V, Röver C and Christensen N 2009 Degeneracies in sky localization determination from a spinning coalescing binary through gravitational wave observations: a Markov-chain Monte Carlo analysis for two detectors Class. Quantum Grav. 26114007 (2009)

[33] van der Sluys M V, Röver C, Stroeer A, Raymond V, Mandel I, Christensen N, Kalogera V, Meyer R and Vecchio A 2008 Gravitational-wave astronomy with inspiral signals of spinning compact-object binaries Astrophys. J. 688 L61-4

[34] Veitch J and Vecchio A 2010 Bayesian coherent analysis of in-spiral gravitational wave signals with a detector network Phys. Rev. D 81062003 
[35] Aasi J et al (LIGO-Virgo Scientific Collaboration) 2013 Parameter estimation for compact binary coalescence signals with the first generation gravitational-wave detector network Phys. Rev. D 88062001

[36] Christensen N, Dupuis R J, Woan G and Meyer R 2004 Metropolis-Hastings algorithm for extracting periodic gravitational wave signals from laser interferometric detector data Phys. Rev. D 70022001

[37] Umstätter R, Meyer R, Dupuis R J, Veitch J, Woan G and Christensen N 2004 Estimating the parameters of gravitational waves from neutron stars using an adaptive MCMC method Class. Quantum Grav. 21 S1655-65

[38] Clark J, Heng I S, Pitkin M and Woan G 2007 Evidence-based search method for gravitational waves from neutron star ring-downs Phys. Rev. D $\mathbf{7 6} 043003$

[39] Summerscales T Z, Burrows A, Finn L S and Ott C D 2008 Maximum entropy for gravitational wave data analysis: recovering the physical parameters from core-collapse supernovae Astrophys. J. $6781142-57$

[40] Heng I S Rotating stellar core-collapse waveform decomposition: a principal component analysis approach Class. Quantum Grav. 26105005

[41] Röver C, Bizouard M-A, Christensen N, Dimmelmeier H, Heng I S and Meyer R 2009 Bayesian reconstruction of gravitational wave burst signals from simulations of rotating stellar core collapse and bounce Phys. Rev. D 80102004

[42] Dimmelmeier H, Ott C D, Marek A and Janka H-T 2008 The gravitational wave burst signal from core collapse of rotating stars Phys. Rev. D 78064056

[43] Logue J, Ott C D, Heng I S, Kalmus P and Scargill J H C 2012 Inferring core-collapse supernova physics with gravitational waves Phys. Rev. D 86044023

[44] Skilling J 2006 Nested sampling for general Bayesian computation Bayes. Anal. 1 833-60

[45] Abdikamalov E, Gossan S, DeMaio A M and Ott C D 2013 Measuring the angular momentum distribution in core-collapse supernova progenitors with gravitational waves Preprint arXiv:1311.3678v1[astro-ph.SR]

[46] Turin G L 1960 An introduction to matched filters IRE Transactions on Information Theory 6 $311-29$

[47] Ott C D, Abdikamalov E, O'Connor E, Reisswig C, Haas R, Kalmus P, Drasco S, Burrows A and Schnetter E 2012 Correlated gravitational wave and neutrino signals from general-relativistic rapidly rotating iron core collapse Phys. Rev. D 86024026

[48] Woosley S E and Heger A 2007 Nucleosynthesis and remnants in massive stars of solar metallicity Phys. Rep. 442 269-83

[49] Lattimer J M and Swesty F D 1991 A generalized equation of state for hot, dense matter Nucl. Phys. A $535331-76$

[50] Shen H, Toki H, Oyamatsu K and Sumiyoshi K 1998 Relativistic equation of state of nuclear matter for supernova and neutron star Nucl. Phys. A 637 435-50

[51] Oppenheim A V, Schafer R W and Buck J R 1999 Discrete-Time Signal Processing (New Jersey: Prentice Hall)

[52] Spiegelhalter D J, Best N G, Carlin B P and van der Linde A 2002 Bayesian measures of model complexity and fit J. R. Statist. Soc. B 64 583-639

[53] Ripley B D 1996 Pattern Recognition and Neural Networks (Cambridge: Cambridge University Press)

[54] Edwards M C, Meyer R and Christensen N 2013 Surfing through the universe: Bayesian inference of gravitational waves 33rd International Workshop on Bayesian Inference and Maximum Entropy Methods in Science and Engineering (Canberra) Poster presentation: https://dcc.ligo.org/DocDB/0110/G1301318/005/MaxEnt13MattEdwards.pdf

[55] Green P J 1995 Reversible jump Markov chain Monte Carlo computation and Bayesian model determination Biometrika 82 711-32

[56] Umstätter R, Christensen N, Hendry M, Meyer R, Simha V, Veitch J, Vigeland S and Woan G 
2005 Bayesian modelling of source confusion in LISA data Phys. Rev. D 72022001 